\title{
Abstract Author Index 32nd Annual Meeting of the Engineering and Urology Society by abstract number
}

A

ABBOUD R, 40, 45

ABERN M, 4

ALARCON G, 22

ALDOUKHI AH, 23, 48, 49

ALJADAAN A, 15

ALLAF ME, 11, 42

ALMALLAH Z, 38

ANASTOS H, 13, 39

ANDRADE C, 22

ANDRADE E, 22

AUTORINO R, 31

AVERCH T, 18

\section{B}

BAILEY M, 47

BALANI J, 9

BARROS AA, 31

BARTLETT A, 9

BASTAWROS DA, 5

BIERWILER H, 43

BILEN C, 17

BLUM KA, 3, 39

BOX G, 18

BRAND T, 18

BRAXTON V, 24

BRISBANE $\mathrm{W}, 47$

BULAI C, 8

BUZZY C, 40, 45

C

CAI K, 4

CALDWELL B, 4

CANALES BK, 46

CANFIELD SE, 13

CARRICK MR, 13

CHANG C-H, 5

CHANG T, 12

CHANG TC, 19

CHENG Z, 12

CHI T, 1, 27

CICCONE JM, 3

CLAYMAN RV, 14, 15, 29, 36, 43

COMSTOCK B, 18

CRUZ A, 29

D

DO KX, 4

DAUN M, 29

DE S, 24

DIAS L, 22
DO R, 44

DOLAN B, 43

DUARTE ARC, 31

E

EBEL J, 10

ENE C, 8

ENE C, 7, 35

ERGEN A, 17

ESFENDIARY T, 46

ESPINO-GROSSO P, 46

F

FABBRO DD, 43

FARAHVASH A, 17

FARHAN B, 44

FASO A, 38

FERRANDINO M, 18

FLEISCHMAN S, 17

FLOWER K, 9

FRANCIS J, 40

FRIED MN, 5, 33

FUNG G, 6

G

GAMBOA AJ, 43

GEAVLETE B, 7, 8, 35

GEAVLETE P, 7, 8, 35

GEORGESCU D, 7, 35

GERMANI S, 43

GHANI KR, 23, 48, 49

GHONIEM G, 16, 44

GLOVER M, 45

GOH A, 37

GOLDSTEIN I, 41

GONZALEZ DA, 33

GORIN MA, 11, 42

GRAVELLE K, 47

GUDELOGLU A, 17

GULANI V, 40

GURNANI R, 6

H

HALL TL, 23, 48, 49

HAN M, 34

HANATA I, 28

HARDY LA, 5, 33

HASEBE T, 28

HELMY M, 29

HERRELL SD, 24

HIDAS G, 20 
HIDAS Y, 20

HIDAS G, 20

HIMESH G, 37

HIRAIWA S, 28

HOANG T, 44

HOFFMANN R, 22

HSI R, 24

HUO Y, 24

HUTCHINSON R, 9, 26

HWANG C, 14

HWANG JH, 47

\section{I}

IARED E, 22

IGEL T, 26

IORDACHE V, 7, 35

IRBY PB, 33

ISAACSON D, 1, 27

IWASAKI M, 32

J

JAMES RC, 15

JECU M, 8

JUN C, 6, 11, 34, 42

K

KALER K, 43

KALER KS, 14

KAOUK J, 18

KAOUK JH, 30

KAWAKAMI M, 32

KAWAMATO S, 6

KENNELLY MJ, 5

KHAN TY, 37

KHOKHLOVA TD, 47

KHOKHLOVA V, 47

KISS B, 12

KLOPFER M, 14

KNAUER CJ, 13, 39

KNUDSEN B, 18

KNUDSEN BE, 10

KOCJANCIC E, 38

KOWALEWSKI TM, 18

L

LADUMOR G, 25, 50

LAMA D, 14

LAMA DJ, 9, 15, 29, 36, 43

LANDMAN B, 24

LANDMAN J, 9, 14, 15, 18, 29, 36,43

LAUGHERY MW, 15

LEE BR, 18

LEE TK, 9

LENDVAY SJ, 18

LEWIS SC, 13, 39

LI P, 6, 11, 42

LIAO J, 19

LIAO JC, 12

LILES WC, 47

LIM S, 6, 11, 34, 42

LIMA E, 31

LIU C, 12

LUCIANO C, 38
LUDWIG W, 6

LUDWIG WW, 42

M

MACH KE, 12

MAHRAN A, 40, 45

MAR W, 4

MARCQ G, 12

MARGULIS V, 9

MATLAGA B, 6

MAURICE MJ, 30

MCDONALD TM, 9

MCDOUGALL E, 18

MENHADJI A, 18

METZLER I, 1

MIANO R, 43

MICALI S, 43

MILLER N, 24

MIYAJIMA A, 28, 32

MOLDOVEANU C, 8

MOREIRA DM, 4

MULTESCU R, 7, 35

MYERS EM, 5

$\mathbf{N}$

NAKANO M, 28, 32

NG J, 37

NGUYEN C, 46

NGUYEN D, 44

NGUYEN N, 16, 44

NIEDERBERGER C, 38

O

OGAWA T, 28, 32

OKHUNOV Z, 9, 14, 29, 43

OLIVEIRA C, 31

OO MM, 37

$\mathbf{P}$

PALMER C, 16, 44

PAN Y, 12

PARTIN AW, 21

PASSEROTTI C, 22

PATEL R, 15

PATEL RM, 14, 36

PATNI LG, 25, 50

PECK AB, 46

PETERS MG, 5

PETRISOR D, 11, 34

PFANNER P, 4

PIERORAZIO PM, 42

PILLARISETTY V, 47

PONSKY L, 40, 45

\section{$\mathbf{R}$}

RASTINEHAD AR, 13, 39

RAWANDALE-PATIL AV, 25, 50

REESE J, 30

REIS RL, 31

RIBEIRO AJ, 31

ROBERTS WW, 23, 48, 49

ROUSE R, 12

ROWE SP, 11

RUBIN D, 19 
RUBIN RS, 41

RUDD A, 29

S

SAFIULLAH S, 9, 14, 15, 29, 36, 43

SAVAGE $\mathrm{S}, 9$

SCARBERRY K, 40

SCHADE GR, 47

SCHAFFHAUSEN C, 18

SCHWARTZ BF, 18

SCHWARTZ JA, 13

SCHWARTZ MJ, 43

SCHWEN ZR, 21

SHAH PH, 43

SHERER B, 27

SHERER BA, 1

SHETH K, 9

SHOJI S, 28, 32

SHUKLA PA, 13, 39

SLAVO L, 22

SOURIAL MW, 10

SOZEN S, 17

SPRADLING K, 9

STANESCU F, 8

STERN JM, 2, 13

STOIANOVICI D, 6, 11, 34, 42

STOLLER M, 27

STOLLER ML, 1

SWEET R, 18

T

TAGUCHI K, 1, 27

TAI CK, 37

TAIN R-W, 4

TAJIRI T, 28

TAOULI B, 39

TAOULI BA, 13

TEWARI AK, 13, 39
THAWANI R, 45

TOMOE H, 32

TORRES-ANGUIANO JR, 38

TZOU D, 27

TZOU DT, 1

$\mathbf{U}$

UCHIDA T, 28

USAWACHINTACHIT M, 1, 27

V

VOIGT A, 46

W

WANCHOO A, 46

WANG M, 45

WANG Y-N, 47

WHANG S, 47

WINOKER JS, 13, 39

WINTER AG, 41

$\mathbf{x}$

XIE K, 4

Y

YANG A, 29

YI D, 19

YOON R, 14, 43

YOUSEFF R, 9

Z

ZAKOJI H, 28, 32

ZHANG L, 44

ZHOU TC, 2

ZIEMBA J, 6

ZIEMBA JB, 42

ZORDANI A, 43 